\title{
Major Radiologic and Clinical Outcomes of Total Spine MRI Performed in the Emergency Department at a Major Academic Medical Center
}

\author{
(D) C.W.C. Huang, (D) A. Ali, (D) Y.-M. Chang, (D)A.F. Bezuidenhout, (D) D.B. Hackney, (D).A. Edlow, and (D).A. Bhadelia
}

\begin{abstract}
BACKGROUND AND PURPOSE: Total spine MRIs are requested by the emergency department when focused imaging can not be ordered on the basis of history or clinical findings. However, their efficacy is not known. We assessed the following: 1) major radiologic and clinical outcomes of total spine MR imaging performed by the emergency department, and 2) whether the presence of a high-risk clinical profile and/or neurologic findings impacts the clinical outcomes.
\end{abstract}

MATERIALS AND METHODS: Total spine MRIs requested by the emergency department during a 28-month period were evaluated for major radiologic (cord compression, cauda equina compression, and other significant findings) and major clinical outcomes (hospital admission during the visit followed by an operation, radiation therapy, or intravenous antibiotics or steroids). Associations between a high-risk clinical profile (cancer, infection, coagulopathy) and/or the presence of neurologic findings and outcomes were assessed.

RESULTS: After we excluded trauma or nondiagnostic studies, 321/2047 (15.7\%) MRIs ordered during study period were total spine MR imaging; $117 / 321$ (36.4\%) had major radiologic and 60/321 (18.6\%) had major clinical outcomes (34/60 in $<24$ hours); and 58/117 (49.6\%) with major radiologic outcome were treated compared with $2 / 205(1.0 \%)$ without $(\mathrm{OR}=99, P<.001)$. The presence of both a high-risk clinical profile and neurologic findings concurrently in a patient (142/321) increased the likelihood of major clinical outcomes during the same visit $(\mathrm{OR}=3.1, P<.001)$ and in $<24$-hours $(\mathrm{OR}=2.6, P=.01)$ compared with those with either a high-risk clinical profile or neurologic findings alone (179/321).

CONCLUSIONS: Total spine MR imaging ordered by our emergency department has a high radiologic and significant clinical yield. When a high-risk clinical profile and neurologic findings are both present in a patient, they should be prioritized for emergent total spine MR imaging, given the increased likelihood of clinical impact.

ABBREVIATIONS: $\mathrm{CEC}=$ cauda equina compression; $\mathrm{ED}=$ emergency department; HRCP $=$ high-risk clinical profile; SCC spinal cord compression; TS = total spine

T wenty-four-hour availability of MR imaging in the United States has significantly increased its use in spine imaging. ${ }^{1-6}$ Although this may be beneficial in many instances, overuse of imaging has become a concern of both hospital administrators and health care policy makers. ${ }^{1,7}$ This concern is based on previous studies that have shown that emergent MR imaging of the spine rarely results in an immediate clinical intervention. ${ }^{1}$ Furthermore, it has also been shown that

Received January 20, 2020; accepted after revision March 24.

From the Departments of Radiology (C.W.C.H., A.A., Y.-M.C., A.F.B., D.B.H., R.A.B.) and Emergency Medicine (J.A.E.), Beth Israel Deaconess Medical Center, Harvard Medical School, Boston, Massachusetts; Department of Radiology (C.W.C.H.), Wan Fang Hospital, Taipei Medical University, Taipei, Taiwan; and Department of

Radiology (C.W.C.H.), School of Medicine, College of Medicine, Taipei Medical University, Taipei, Taiwan.

Please address correspondence to Rafeeque A. Bhadelia, MD, Department of Radiology, Beth Israel Deaconess Medical Center, WCB90, 330 Brookline Ave, Boston, MA, 02215; e-mail: rbhadeli@bidmc.harvard.edu; @rbhadeliaMD

http://dx.doi.org/10.3174/ajnr.A6578
MR imaging of the lumbar spine does not improve overall clinical outcome compared with standard clinical care in patients without "red flags" indicating underlying conditions such as cancer, infection, or cauda equina syndrome. ${ }^{8}$

Because clinical symptoms and physical examination are often inconsistent and inadequate in identifying and localizing spinal pathology in patients presenting with neck or back pain and neurologic deficits, ${ }^{4,9-11}$ MR imaging is frequently ordered for patients in the emergency department (ED). Despite increasing regulatory scrutiny from governmental and private insurers, over-reliance on expensive imaging studies in this clinical scenario is often justified because delayed diagnosis and treatment of spinal cord or cauda equina compression (CEC) may result in permanent morbidity or even mortality. ${ }^{10-13}$

While most major radiology departments with 24/7 MR imaging service are able to accommodate unscheduled emergent singlesegment spine MR imaging ordered by the $\mathrm{ED}$, an unscheduled 
total spine (TS) MR imaging can disrupt the normal workflow due to longer scan and interpretation times compared with a singlelevel study and would likely reduce its use. ${ }^{14}$ It is generally understood that TS-MR imaging is ordered by the ED when patients have a high-risk clinical profile (HRCP) such as a history of cancer and clinical suspicion of infection or coagulopathy and when distinction between spinal cord and cauda equina compression could not be made clinically. ${ }^{11,14}$ Except for a single study showing extremely low radiologic and clinical outcomes of TS-MR imaging for spinal cord compression, ${ }^{14}$ there are insufficient data about the clinical impact of emergent TS-MR imaging examinations ordered by the ED to assess the appropriateness of these studies or implement change in use. Therefore, our purpose was to assess the major radiologic and clinical outcomes of TS-MR imaging performed by the ED and whether HRCP and/or neurologic findings impact the clinical outcome.

\section{MATERIALS AND METHODS Patient Population}

The patient population was drawn from a major academic medical center (Beth Israel Deaconess Medical Center, Boston, Massachusetts) serving a large metropolitan city and surrounding area, with 704 hospital beds. The hospital is a level 1 trauma and comprehensive cancer, stroke, neurosurgical, and spine center with approximately 56,000 ED visits per year.

Institutional review board approval was obtained for this retrospective study of adult (18 years of age or older) patients with a waiver of informed consent. This study was based on imaging data collected for a quality-assurance project designed to assess use of emergent spinal MRIs obtained by the ED at our institution between March 1, 2016, and June 30, 2018. The inclusion criterion was a TS-MR imaging examination ordered by the ED. Exclusion criteria were a history of major trauma or a nondiagnostic examination. Repeat examinations performed on the same day for either additional contrast-enhanced images or a nondiagnostic, inconclusive, or incomplete initial study were considered 1 examination.

During the study period, 384 consecutive TS-MR imaging examinations were performed of 2047 spine MRIs ordered from the ED. We excluded 63/384 TS-MRIs: 46/384 for a history of major trauma and 17/384 for examinations being nondiagnostic either due to severe motion artifacts or pain or claustrophobia (that were not repeated later). Examinations performed for major trauma were excluded because most of those studies were performed for evaluation of abnormalities seen on an initial CT examination or due to severe neurologic findings and thus would have a bias for an increased pretest probability of major clinical outcomes. Thus, 321 TS-MR imaging examinations were included in the study.

\section{MR Imaging}

The MR imaging examinations were performed on either a $1.5 \mathrm{~T}$ or 3T Signa HDx scanner (GE Healthcare) or a 1.5T Magnetom Espree scanner (Siemens). All MR imaging was performed with a standard protocol, including sagittal T1-weighted, T2-weighted, and inversion recovery and axial T2 images through the entire spine. The sagittal images (3-mm thickness; FOV $=34 \mathrm{~cm}$; matrix $=384-$ $512 \times 256$ ) covered the entire spine in 2 acquisitions (posterior fossa to T6-7 and T5-6 to S2-3 levels) and axial contiguous images (5$\mathrm{mm}$ thickness; $\mathrm{FOV}=20 \mathrm{~cm}$; matrix $=256 \times 256)$ in 4 acquisitions (cervical, upper thoracic, lower thoracic, and lumbar). If gadolinium was administered, then T1-weighted sagittal and axial postcontrast images were also obtained (230/321 patients undergoing TS-MR imaging received gadolinium). Pregadolinium T1-weighted axial images through the spine were not obtained, to limit the imaging time, which was $45-50$ minutes without and 65-70 minutes with gadolinium for a TS-MR imaging examination.

\section{Radiologic Outcomes}

TS-MR imaging was considered to have a major radiologic outcome with $\geq 1$ of the following findings:

- Spinal cord compression (SCC): severe spinal canal narrowing with compression of the spinal cord and a lack of surrounding CSF (Fig 1). ${ }^{15}$

- Cauda equina compression: severe narrowing of the lumbar spinal canal on axial images and lack of CSF within the thecal $\operatorname{sac}\left(\right.$ Fig 2). ${ }^{16}$

- Other significant findings: findings without spinal cord or cauda equina compression that may affect clinical management (Fig 3), such as an intramedullary spinal cord lesion (other than myelomalacia), spinal and extraspinal manifestations of infection (discitis/osteomyelitis and abscess), bone or soft-tissue abnormalities (fracture, hematoma, lymphadenopathy, mass), or vascular abnormalities.

Degenerative spinal stenosis or disc herniation without SCC or CEC and the presence of neural foraminal narrowing were not considered major radiologic outcomes for TS-MR imaging.

\section{Clinical Outcomes}

TS-MR imaging was considered to have a major clinical outcome if the patient was admitted and treated with an operation, radiation, intravenous antibiotics, or steroid therapy or image-guided abscess drainage during the same visit on the basis of the radiologic findings. If a patient received empiric IV antibiotics or steroid therapy while in the ED but these treatments were not performed on the inpatient service, it was not considered a major clinical outcome. The clinical outcomes were further refined to identify those who received treatment within the first 24 hours of arrival to the ED.

\section{History and Neurologic Findings}

Electronic medical records of patients including ED and specialist consultation notes (if available) were reviewed and stratified as having the presence or absence of HRCP and neurologic findings accordingly.

Patients were considered to have a HRCP when a history of cancer, predisposing factors, or evidence for infection (back pain with fever, intravenous drug use, bacteremia, or laboratory markers of infection), coagulopathy, prior spinal intervention, or demyelinating disease was noted in their history.

Patients were considered to have positive neurologic findings if there was $\geq 1$ of the following present: bilateral extremity pain or weakness, sensory deficits, abnormal reflexes, urinary or stool incontinence, decreased anal tone, and/or saddle anesthesia. 

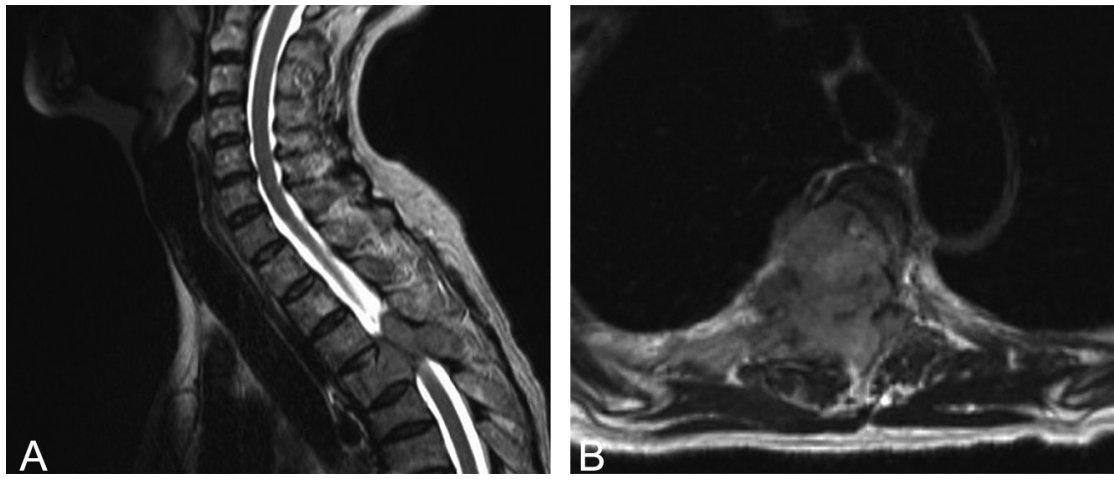

FIG 1. A 67-year-old woman with a history of breast cancer presented with bilateral lower extremity and right upper extremity weakness. The upper portion of total spine MR imaging with T2-weighted sagittal $(A)$ and axial $(B)$ images shows severe compression of the spinal cord at the T4 level with no CSF visualized within the spinal canal at that level.
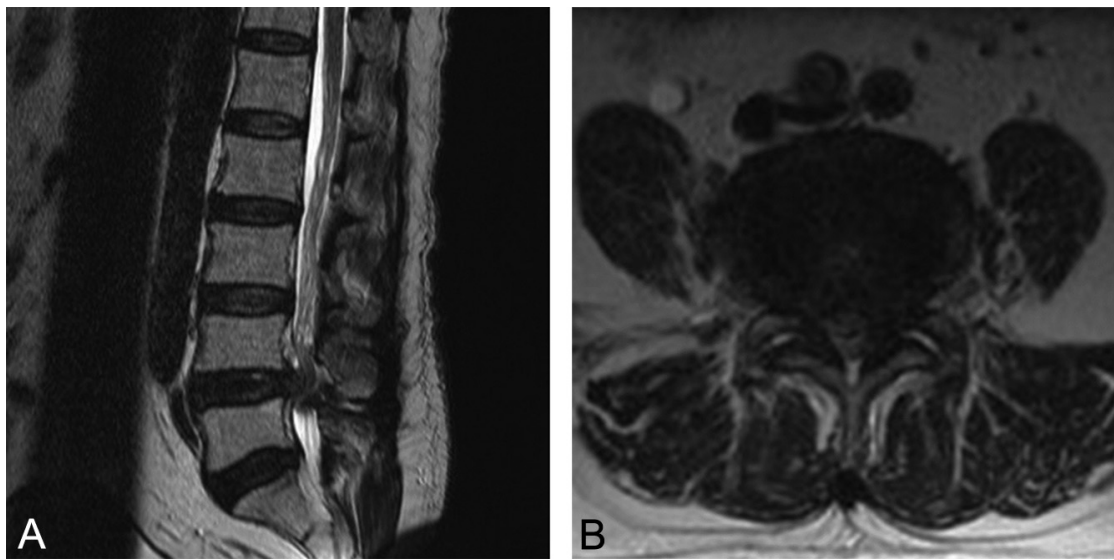

FIG 2. An 83-year-old man presenting with lower back pain, leg numbness, leg weakness, and urinary retention. T2-weighted sagittal $(A)$ and axial $(B)$ images from total spine MR imaging show a herniated L4-L5 intervertebral disc causing severe spinal canal stenosis with a lack of CSF within the thecal sac, suggesting cauda equina compression.
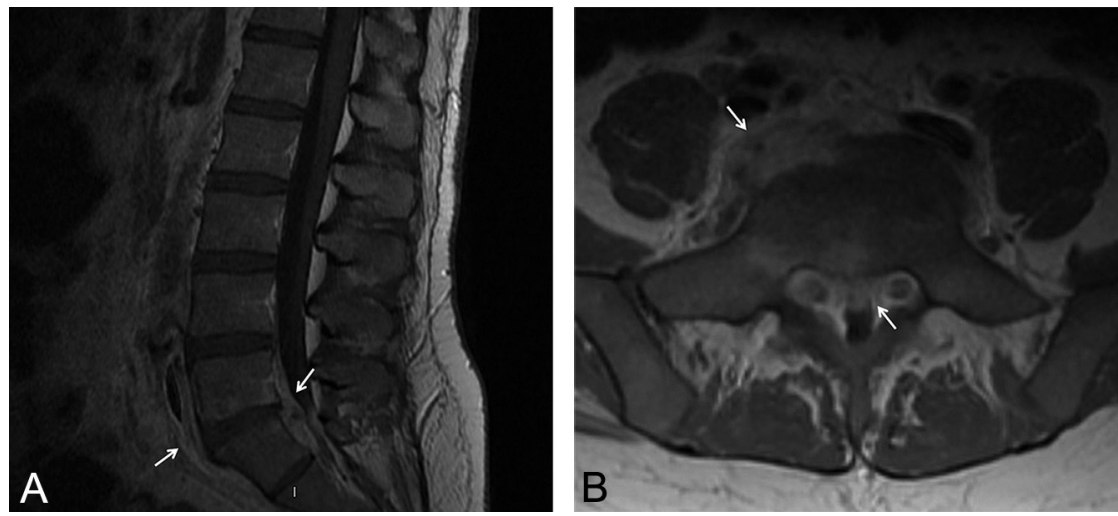

FIG 3. A 41-year-old man with a history of intravenous drug use presenting with fever, severe diffuse back pain, and right lower quadrant abdominal pain. The lower portion of total spine MR imaging with Tl-weighted postcontrast sagittal $(A)$ and axial $(B)$ images shows discitis and osteomyelitis, with epidural and right paraspinal phlegmon.

\section{Data Analysis}

The presence and absence of radiologic and clinical outcomes were recorded. Patients who had major radiologic outcome at $>1$ spinal segment (cervical, thoracic, or lumbar spine) were considered to have multisegment disease. The radiologic outcomes among SCC, CEC, or other significant findings that were treated or considered most clinically symptomatic (from medical records review) were included in the analysis.

Odds ratios were determined to assess the strength of association between the following:

- Major clinical outcomes between patients with and without major radiologic outcomes.

- Major clinical outcomes between patients who had both HRCP and neurologic findings and those who had either HRCP or neurologic findings alone.

A radiologic or clinical outcome $\geq 5 \%$ was considered a significant outcome. An on-line calculator (https://www.medcalc. org/calc/odds_ratio.php) was used to determine OR, 95\% CI, and significance. $P<.05$ was considered significant.

\section{RESULTS}

After exclusions for trauma or nondiagnostic studies, 321/2047 (15.7\%) MR imaging examinations ordered during the study period by the ED were TSMR imaging. The mean age of 321 patients was $52 \pm 17$ years; 171/321 (53\%) were women (52 \pm 17 years) and 150/321 (47\%) were men (51 \pm 17 years).

\section{Radiologic Outcomes}

Table 1 shows radiologic outcomes of 321 TS-MR imaging examinations; $117 / 321$ (36\%) had a major radiologic outcome, and 204/321 (64\%) had no major radiologic outcome. Forty-eight of 117 (41\%) patients with radiologic outcomes had major findings at multiple spinal segments, and 69/117 (59\%) had major findings at single spinal segment.

SCC or CEC was noted in $72 / 321$ (22.4\%) TS-MR imaging examinations. The most common cause for SCC or CEC was degenerative disease 


\begin{tabular}{|c|c|c|c|}
\hline \multirow{2}{*}{$\begin{array}{l}\text { Radiologic Outcomes of TS-MR Imaging } \\
\text { with Major Radiologic Outcome }\end{array}$} & \multirow{2}{*}{$\begin{array}{c}(N=321) \\
117 / 321(36.4 \%)\end{array}$} & \multicolumn{2}{|c|}{ Major Clinical Outcome in Patients, $(n=60 / 321)(18.6 \%)$} \\
\hline & & During Visit 58/117 (49.6\%) ${ }^{\mathrm{b}}$ & $<24$ Hours $33 / 117(28.2 \%)^{\circ}$ \\
\hline SCC or CEC & 72 & 36 & 20 \\
\hline Degenerative disease & 41 & 12 & 3 \\
\hline Neoplasm & 16 & 12 & 5 \\
\hline Infection & 10 & 10 & 10 \\
\hline Hematoma & 2 & 2 & 2 \\
\hline latrogenic & 1 & 0 & 0 \\
\hline Traumatic findings & 2 & 0 & 0 \\
\hline Other significant findings & 45 & 22 & 13 \\
\hline Neoplasm & 19 & 8 & 1 \\
\hline Infection & 8 & 8 & 8 \\
\hline latrogenic & 1 & 1 & 1 \\
\hline Spinal cord lesion & 7 & 3 & 3 \\
\hline Traumatic findings & 3 & 0 & 0 \\
\hline Extraspinal findings ${ }^{d}$ & 7 & 2 & 0 \\
\hline Without major radiologic outcome & $204 / 321(63.6 \%)$ & $2 / 204(1.0 \%)^{b}$ & $1 / 204(0.5 \%)^{c}$ \\
\hline
\end{tabular}

Note:-Bold indicates total of subsequent raws respectively.

${ }^{a}$ Clinical Outcome: hospital admission followed by an operation, radiation, IV therapy (antibiotics or steroids), or abscess drainage during same visit as well as in $<24$ hours and $>24$ hours after arrival to ED.

${ }^{\mathrm{b}}$ Odds Ratio $=99$ (95\% Cl, 23.5-419; $P$ value $\left.<.001\right)$ between patients with and without major radiologic outcomes.

${ }^{c}$ Odds Ratio $=80$ (95\% Cl, 10.7-593; $P$ value $\left.<.001\right)$ between patients with and without major radiologic outcomes.

'Psoas muscle abscess and lymphoma.

Table 2: Impact of HRCP and neurologic findings on major clinical outcomes ${ }^{a}$

\begin{tabular}{lllcccc}
\hline \multicolumn{1}{c}{ Variable } & No. of Patients & Odds Ratio & & & Odds Ratio \\
$(\mathbf{N}=321)$ & During Visit & $(95 \% \mathrm{Cl})$ & $\boldsymbol{P}$ Value & $<24$ Hours & $\boldsymbol{P}$ Value \\
\hline HRCP + NF & $142 / 321(44 \%)$ & $40 / 142(28 \%)$ & $3.1(1.7-5.6)$ & $<.001$ & $22 / 142(15 \%)$ & $2.6(1.2-5.4)$ \\
HRCP or NF alone & $179 / 321(56 \%)$ & $20 / 179(11 \%)$ & & & $12 / 179(7 \%)$ & .01 \\
\hline
\end{tabular}

Note:-NF indicates neurologic findings.

${ }^{a}$ Major clinical outcomes of patients who had both HRCP + NF were compared with patients who had either HRCP or NF alone.

(57\%), followed by neoplasm (22\%) and infection (14\%). Other significant findings were noted in 45/321 (14\%) TS-MR imaging examinations. The most common cause of other significant findings was neoplasm (42\%), followed by infection (18\%), spinal cord lesion (15\%), and extraspinal findings (15\%).

\section{Clinical Outcomes}

Table 1 shows major clinical outcomes of 321 TS-MR imaging examinations; 60/321 (19\%) had major clinical outcome, and 34/ 321 (11\%) were treated within 24 hours of arrival in the ED. Patients with a major radiologic outcome were more likely to have a major clinical outcome (58/117) compared with those without a major radiologic outcome $(2 / 204, \mathrm{OR}=99 ; 95 \% \mathrm{CI}, 23.5-419 ; P<$ .001). The patients with a major radiologic outcome were also more likely to have a $<24$-hour clinical outcome compared with those without one (OR $=80$; 95\% CI, 10.7-593; $P<.001)$.

Of the 16/72 patients with SCC/CEC due to tumor, 12/16 (75\%) had a major clinical outcome, 9/12 patients underwent an operation ( 4 in $<24$ hours), and $3 / 12$ underwent radiation (1 in $<24$ hours). Of the 41 patients with SCC/CEC due to degenerative disease, 12/41 (29\%) had major clinical outcome (3 in $<24$ hours), and they underwent an operation. Of the 19 patients with other significant findings (without SCC/CEC) for neoplasm, 2/19 underwent an operation ( 1 in $<24$-hours) and 6/19 underwent radiation therapy within the same visit.

All 18/18 patients with major radiologic findings for infection (SCC/CEC or other significant findings) had a major clinical outcome. All received IV antibiotic treatment. Seven underwent an operation ( 6 in $<24$ hours), and 2 had image-guided drainage of an associated abscess.

Two of 204 patients without a major radiologic finding underwent an operation. One patient underwent anterior cervical fusion for cervical radiculopathy with severe foraminal narrowing at the C5-C6 level with moderate spinal stenosis without cord compression or cord signal abnormality. The second patient underwent revision laminectomy with far lateral decompression and spinal fusion at the L3-L4 levels for radiculopathy.

\section{Impact of HRCP and/or Neurologic Findings on Clinical Outcomes}

Table 2 shows the impact of HRCP and neurologic findings on major clinical outcomes.

One hundred forty-two of 321 (44\%) patients presented with both HRCP and neurologic findings compared with 179/321 (56\%) patients who had either HRCP or neurologic findings alone (80/179 with HRCP alone and 99/179 with neurologic findings alone). There were no patients without HRCP or neurologic findings who underwent TS-MR imaging.

The likelihood of a major clinical outcome during an ED visit (admission followed by an operation, radiation, intravenous antibiotics or steroids therapy, or image-guided abscess drainage) was significantly higher in patients with both HRCP and neurologic findings than in those with either HRCP or neurologic findings alone $(\mathrm{OR}=3.1 ; 95 \% \mathrm{CI}, 1.7-5.6 ; P<.001)$. Finally, the likelihood 
of $<24$-hour clinical outcome after an ED visit was also increased in those with both HRCP and neurologic findings compared with those with either HRCP or neurologic findings alone $(\mathrm{OR}=2.6$; $95 \% \mathrm{CI}, 1.2-5.4 ; P=.01)$.

\section{DISCUSSION}

Our results suggest that TS-MR imaging performed by the ED at a tertiary care academic medical center has a high radiologic yield, with major findings seen in 1 of 3 patients despite using stringent criteria for a radiologic outcome with positive findings. Furthermore, although major clinical outcomes of these studies were lower than radiologic outcomes, they were not insignificant. Nearly 1 of 6 patients imaged were treated during the same hospital visit, and nearly 1 of 10 imaged was treated within the first 24 hours of arrival in the ED. We also observed that the clinical yield of TS-MR imaging was much higher in patients who had both HRCP and neurologic findings simultaneously than in either of them alone. Moreover, those with SCC/CEC due to tumor/infection were more likely to be treated than those with degenerative disease. Finally, an interesting additional finding of the study was that the use of strict radiologic criteria improves the clinical impact of radiologic observation, with nearly half of the patients with positive radiologic outcomes receiving treatment during the hospital visit. On the basis of these results, we recommend that for interpretation of TS-MR imaging studies, strict diagnostic criteria be used to improve their clinical impact. ${ }^{17,18}$

American College of Radiology recommendations suggest that performing emergent MR imaging of the spine is appropriate in the setting of back pain associated with red flags such as cancer, infection, coagulopathy, or cauda equina syndrome. ${ }^{5}$ Similarly, many previous studies have also used signs and symptoms in the patient's history and clinical examination in combination as red flags. ${ }^{6,8}$ In this study, we took a slightly different approach and used a patient's high-risk clinical profile (such as history of cancer, predisposing factors, or evidence of infection and coagulopathy) and neurologic findings (neurologic signs and symptoms such as bilateral extremity pain or weakness, sensory deficits, abnormal reflexes, urinary or stool incontinence, decreased anal tone, and/or saddle anesthesia) as separate variables to assess their impact on clinical outcomes of TS-MR imaging ordered by the ED. We were able to quantitatively demonstrate that the simultaneous presence of both HRCP and neurologic findings in a patient significantly increased the likelihood of positive clinical outcomes compared with those who had HRCP or neurologic findings alone. Our results thus provide a practical guideline: A patient who has both HRCP and neurologic findings should be prioritized in the ED setting for an emergent TS-MR imaging.

Previous studies evaluating the radiologic and clinical outcomes of spine MR imaging have generally focused on a single-segment spine study such as cervical or lumbar spine MR imaging. ${ }^{1,19}$ Black et $\mathrm{al}^{1}$ looked at the radiologic and clinical outcomes of 179 emergent spine MRIs during a 13-year period. There were 77 cervical, 87 thoracic, and 101 lumbar spine MR imaging studies, in combination or in isolation, without providing a specific number for TS-MR imaging. A significant radiologic finding was seen in 104/179 (58\%), and 66/179 (36.8\%) were treated within 48 hours. ${ }^{1}$ Another study by Gardner et $\mathrm{al}^{19}$ looking only at the lumbar spine for patients with cauda equina syndrome found that $33 \%$ of patients with suspected cauda equina syndrome had positive radiologic findings but only $7 \%$ received treatment. Both of these studies used different radiologic outcome criteria and different patient populations such as those with mixed single- and multiple-segment MR imaging scans as in the study by Black et al and lumbar spine studies in case of Gardner et al compared with all TS-MR imaging in our study, making it difficult to compare the results. Nevertheless, our results are similar to those previous studies when accounting for these differences.

However, our results are quite dissimilar to those by Potigailo et $\mathrm{al}^{14}$ who found a very low $1.4 \%$ radiologic outcome of total spine MR imaging performed by the ED for acute spinal cord compression, which increased to $4.4 . \%$ after an institutional change in policy of ordering spine MR imaging for spinal cord compression. Unlike their study, in our study, 22.4\% (72/321) of TS-MR imaging was positive for spinal cord or cauda equina compression despite using a rather restrictive definition for both the entities. The reason for the high radiologic outcomes in our study appears to be that our ED ordered TS-MR imaging only when spine-related symptoms were associated with a high-risk clinical profile or neurologic findings, and none of the studies were ordered for vague symptoms.

An emergent TS-MR imaging is challenging to a radiology department both for performance and interpretative purposes and from the health care policy perspective due to its added cost. A TS-MR imaging takes between 60 and 75 minutes of MR imaging scan time (total MR imaging table time) compared with 2535 minutes for a single-segment spine MR imaging or brain MR imaging. Furthermore, most of these studies ordered from the ED are emergent and need to be performed immediately to avoid delays in diagnosis and treatment. Because MR imaging scanners at most major academic hospitals are in constant use, a TS-MR imaging is difficult to accommodate, given the time required. There is also a concern about their interpretations around the clock because large numbers of images have to be reviewed, with decreased spatial resolution given the larger FOV per a given matrix size, and these may increase the potential for interpretation errors. Additionally, the cost of obtaining a TS-MR imaging is more than twice that of a single-segment spine MR imaging. In light of the aforementioned, it is important to find ways to optimize the use of TS-MR imaging by developing guidelines for its use, which are currently lacking.

Our data are hypothesis-generating. They have provided a roadmap for a prospective study in collaboration with the ED that may help in reducing the use of TS-MR imaging. First, how successful ED physicians are in restricting the TS-MR imaging orders to a single level using their clinical judgment even in the presence of equivocal findings should be assessed. A prospective study would allow a physician to record the most likely pathologic level while ordering a TS-MR imaging (when they are unable to restrict it to a single level) so that their localizing accuracy can be subsequently assessed. Such a study we believe would be much superior to a retrospective attempt of comparing results of TS-MR imaging and single-level MR imaging and can be done without putting patients at risk of missing a clinically important finding such spinal cord or cauda equina compression. Second, while a history of cancer may not have a quick laboratory test to exclude its presence, infection can be 
detected with a rapid laboratory test such as C-reactive protein. Thus, a prospective study should use estimation of an objective laboratory finding such as C-reactive protein (as opposed to clinical suspicion of infection) to provide an independent proof of the presence of infection before an MR imaging is performed. ${ }^{20-22}$ While a high C-reactive protein level does not always indicate that a spinal infection is present because it is nonspecific and could be positive for infection anywhere in the body, a low level may eliminate the need for an emergent TS-MR imaging study.

There are a few limitations to our study. First, our hospital is a tertiary care center with 24-hour MR imaging availability. Therefore, many patients may have been referred here specifically for further treatment for a process noted on other imaging techniques at an outside institution. This situation may increase the number of positive outcomes. However, as noted previously, this effect was likely minimal because our results are comparable with those of previous studies. Second, our study was a retrospective one, and the clinical information about risk profile and neurologic findings was obtained from the electronic records, which could be missing some important observations made by the clinicians. While TS-MR imaging constituted a small percentage (one-sixth) of all emergent spine MR imaging ordered by the ED during the study period, we were unable to consistently determine from the medical records why a particular patient underwent TS-MR imaging in place of a focused MR imaging study. Likewise, we were not always able to determine the exact time of onset of the patient's symptoms that brought them to the ED and whether their symptoms were a recent exacerbation of chronic symptoms. Therefore, a prospective study is required before an attempt is made to decrease/optimize TS-MR imaging use based on this information. Third, in several cases, documentation of the timing of treatment was unclear and may have affected our results for therapy under 24 hours. Last, it was not possible to assess long-term outcomes of patients after they underwent TS-MR imaging because many patients likely received follow-up assessment elsewhere.

\section{CONCLUSIONS}

We observed a high radiologic yield for TS-MR imaging performed by the ED with clinical outcomes lower but not insignificant. The presence of both a high-risk clinical profile and neurologic findings in a patient increases the likelihood of positive clinical outcomes. We propose that a patient who simultaneously has both a high-risk clinical profile and neurologic findings should be prioritized for emergent TS-MR imaging, given the significantly increased likelihood of a clinical outcome.

Disclosures: David B. Hackney-UNRELATED: Grants/Grants Pending: National Institutes of Health-National Institute of Arthritis and Musculoskeletal and Skin Diseases, Comments: grant to study the risk of vertebral fracture in patients treated with stereotactic body radiosurgery for spinal metastases.* Jonathan A. Edlow-UNRELATED: Expert Testimony: CRICO, Boston, MA. Comments: review of medico-legal cases for both plaintiff and defense firms. ${ }^{*}$ Money paid to the institution.

\section{REFERENCES}

1. Black DF, Wood CP, Wells ML, et al. Emergent, after hours magnetic resonance imaging of the spine. J Neuroimaging 2015;25:59094 CrossRef Medline
2. Pakpoor J, Saylor D, Izbudak I, et al. Emergency department MRI scanning of patients with multiple sclerosis: worthwhile or wasteful? AJNR Am J Neuroradiol 2017;38:12-17 CrossRef Medline

3. Rankey D, Leach JL, Leach SD. Emergency MRI utilization trends at a tertiary care academic medical center: baseline data. Acad Radiol 2008;15:438-43 CrossRef Medline

4. Ahad A, Elsayed M, Tohid H. The accuracy of clinical symptoms in detecting cauda equina syndrome in patients undergoing acute MRI of the spine. Neuroradiol J 2015;28:438-42 CrossRef Medline

5. Lavi ES, Pal A, Bleicher D, et al. MR imaging of the spine: urgent and emergent indications. Semin Ultrasound CT MR 2018;39:55169 CrossRef Medline

6. Sizer PS Jr, Brismee JM, Cook C. Medical screening for red flags in the diagnosis and management of musculoskeletal spine pain. Pain Pract 2007;7:53-71 CrossRef Medline

7. Iglehart JK. Health insurers and medical-imaging policy: a work in progress. N Engl J Med 2009;360:1030-37 CrossRef Medline

8. Chou $\mathrm{R}, \mathrm{Fu} \mathrm{R}$, Carrino JA, et al. Imaging strategies for low-back pain: systematic review and meta-analysis. Lancet 2009;373:463-72 CrossRef Medline

9. Rooney A, Statham PF, Stone J. Cauda equina syndrome with normal MR imaging. J Neurol 2009;256:721-25 CrossRef Medline

10. Gitelman A, Hishmeh S, Morelli BN, et al. Cauda equina syndrome: a comprehensive review. Am J Orthop (Belle Mead NJ) 2008;37:55662 Medline

11. Ropper AE, Ropper AH. Acute spinal cord compression. $N$ Engl J Med 2017;376:1358-69 CrossRef Medline

12. Davis JW, Phreaner DL, Hoyt DB, et al. The etiology of missed cervical spine injuries. J Trauma 1993;34:342-46 CrossRef Medline

13. Ahn UM, Ahn NU, Buchowski JM, et al. Cauda equina syndrome secondary to lumbar disc herniation: a meta-analysis of surgical outcomes. Spine (Phila Pa 1976) 2000;25:1515-22 CrossRef Medline

14. Potigailo VL, Christoforidis GA, Katzman GL. Acute spinal cord compression: CQI framework increases resource efficiency while promoting delivery of high-quality care. J Am Coll Radiol 2015;12:90-94 CrossRef Medline

15. Bilsky MH, Laufer I, Fourney DR, et al. Reliability analysis of the epidural spinal cord compression scale. J Neurosurg Spine 2010;13:32428 CrossRef Medline

16. Huang CWC, Ali A, Chang YM, et al. Performance of on-call radiology residents in interpreting total spine MRI studies for the detection of spinal cord compression or cauda equina compression. $A J R$ Am J Roentgenol 2019;213:1341-47 CrossRef Medline

17. Stafira JS, Sonnad JR, Yuh WT, et al. Qualitative assessment of cervical spinal stenosis: observer variability on CT and MR images. AJNR Am J Neuroradiol 2003;24:766-69 Medline

18. Weber C, Rao V, Gulati S, et al. Inter- and intraobserver agreement of morphological grading for central lumbar spinal stenosis on magnetic resonance imaging. Global Spine J 2015;5:406-10 CrossRef Medline

19. Gardner A, Gardner E, Morley T. Cauda equina syndrome: a review of the current clinical and medico-legal position. Eur Spine J 2011;20:690-97 CrossRef Medline

20. Davis DP, Salazar A, Chan TC, et al. Prospective evaluation of a clinical decision guideline to diagnose spinal epidural abscess in patients who present to the emergency department with spine pain. Neurosurg Spine 2011;14:765-70 CrossRef Medline

21. Davis DP, Wold RM, Patel RJ, et al. The clinical presentation and impact of diagnostic delays on emergency department patients with spinal epidural abscess. J Emerg Med 2004;26:285-91 CrossRef Medline

22. Davis WT, April MD, Mehta S, et al. High risk clinical characteristics for pyogenic spinal infection in acute neck or back pain: prospective cohort study. Am J Emerg Med 2019 May 17. [Epub ahead of print] CrossRef Medline 